\title{
THE HYPERSPACE OF TOTALLY DISCONNECTED SETS
}

\author{
Raúl Escobedo, Patricia Pellicer-Covarrubias and Vicente \\ SÁNCHEZ-GutiérREZ \\ Benemérita Universidad Autónoma de Puebla, México
}

\begin{abstract}
In this paper we study the hyperspace of all nonempty closed totally disconnected subsets of a space, equipped with the Vietoris topology. We show results of compactness, connectedness and local connectedness for this hyperspace. We also include a study of path connectedness, particularly we prove that for a smooth dendroid this hyperspace is pathwise connected, and we present a general result which implies that for an Euclidean space this hyperspace has uncountably many arc components.
\end{abstract}

\section{INTRODUCTION}

In this paper the spaces are $T_{1}$, we mean the one-point sets are closed sets. For a space $X$ we denote by $2^{X}$ the collection of all nonempty closed subsets of $X$, equipped with the Vietoris topology, [8, Definition 1.7] and [10, Definition (0.12)]. A subspace of $2^{X}$ is called a hyperspace of the space $X$. A problem treated by many authors is to investigate topological properties of a space through its hyperspaces and vice versa, see references in the classical books in this matter [5] and [10]. Here we begin the study of the hyperspace of closed and totally disconnected subsets of a space $X$, that we denote by $T D(X)$. After Definitions, in Section 3, we show some basic facts concerning the topological structure of this hyperspace, and we present conditions for compactness, connectedness and local connectedness for it. In Section 4, we prove that for every smooth dendroid $X$ the hyperspace $T D(X)$ is contractible, and we include an example of a (non-smooth) dendroid for which this hyperspace is not pathwise connected. We also prove that for a Hausdorff,

2020 Mathematics Subject Classification. 54B20, 54F15, 54G05.

Key words and phrases. Continuum, hyperspace, locally connected, pathwise connected, totally disconnected set. 
locally compact, not compact and Lindelöf space $X$, the hyperspace $T D(X)$ has uncountably many arc components.

\section{Definitions}

Given a subset $A$ of a space $X$, the interior and the closure of $A$ in $X$ are denoted by $\operatorname{int}(A)$ and $\bar{A}$, respectively. The cardinality of the set $A$ is denoted by $|A|$. We use the symbols $\mathbb{R}$ and $\mathbb{N}$ to denote the set of all real numbers and the set of all positive integers, respectively. Also $|\mathbb{R}|$ es denoted by c. A cellular family in a space $X$ is a family of pairwise disjoint nonempty open subsets of $X$. The collection of all finite cellular families of a space $X$ is denoted by $\mathfrak{C}(X)$. The dimension of a space $X$ is denoted by $\operatorname{dim}(X)$.

A subset $A$ of a space $X$ is totally disconnected provided that no connected subset of $A$ consists of more than one point. We recall that if $p$ is a point of a space $X$, then $X$ is said to be locally connected (connected im kleinen) at $p$ provided that for each open subset $U$ of $X$ such that $p \in U$, there exists a connected open subset (a connected subset) $V$ of $X$ such that $p \in V$ $(p \in \operatorname{int}(V))$ and $V \subset U$. The space $X$ is said to be locally connected provided that it is locally connected at each of its points. It is clear that if a space is locally connected at a point $p$, then it is connected im kleinen at $p$; the converse is false (see the example in [11, Figure 5.22, p. 84]). Nevertheless, it is easy to prove that these two notions are globally equivalent, i.e., a space is locally connected if and only if it is connected im kleinen at each of its points.

For a space $X$, let

$$
\begin{gathered}
2^{X}=\{A \subseteq X: A \text { is closed in } X \text { and } A \neq \emptyset\}, \\
T D(X)=\left\{A \in 2^{X}: A \text { is totally disconnected }\right\}, \text { and } \\
\\
K(X)=\left\{A \in 2^{X}: A \text { is compact }\right\} .
\end{gathered}
$$

Given a finite family of open subsets $U_{1}, \ldots, U_{n}$ of $X$, we define $\left\langle U_{1}, \ldots, U_{n}\right\rangle$ as the subset of $2^{X}$ consisting of those elements $A$ such that $A \subseteq U_{1} \cup \cdots \cup U_{n}$ and $U_{i} \cap A \neq \emptyset$ for each $i \in\{1, \ldots, n\}$. The Vietoris topology is the topology in $2^{X}$ generated by the base consisting of all sets of the form $\left\langle U_{1}, \ldots, U_{n}\right\rangle$ where $n \in \mathbb{N}$ and $U_{i}$ is an open subset of $X$, see [8, Proposition 2.1, p. 155]. The hyperspace $T D(X)$ will be considered as subspace of $2^{X}$. In particular, a base for the topology of $T D(X)$ consists of all sets of the form $\left\langle U_{1}, \ldots, U_{n}\right\rangle \cap$ $T D(X)$.

An arc is a space homeomorphic to the closed interval $[0,1]$. A continuum is a nondegenerate compact connected metric space. A continuum $X$ is unicoherent provided that whenever $A$ and $B$ are closed connected subsets of $X$ such that $X=A \cup B$, then $A \cap B$ is connected; $X$ is hereditarily unicoherent if each subcontinuum of $X$ is unicoherent. A dendroid is a hereditarily unicoherent and arcwise connected continuum. Given different points $a$ and $b$ in a dendroid $X$, the unique $\operatorname{arc}$ in $X$ with end points $a$ and $b$ is denoted 
by $a b$. A dendroid is smooth if there exists some point $p \in X$ such that given any sequence $\left\{a_{n}\right\}_{n \in \mathbb{N}}$ in $X$ with $\lim a_{n}=a$, it holds that $\lim a_{n} p=a p$.

\section{BASIC FACTS, COMPACTNESS, CONNECTEDNESS AND LOCAL CONNECTEDNESS}

In this section we prove some basic facts about interior, compactness, connectedness, local connectedness and compactness of the hyperspace $T D(X)$.

Lemma 3.1. If $A$ is a nonempty compact and totally disconnected subset of a Hausdorff space $X$, then for each finite collection of open subsets of $X$, $V_{1}, \ldots, V_{n}$, such that $A$ belongs to $\left\langle V_{1}, \ldots, V_{n}\right\rangle$, there exist a finite collection of pairwise disjoint nonempty closed subsets of $X, A_{1}, \ldots, A_{k}$, and a finite collection of pairwise disjoint open subsets of $X, U_{1}, \ldots, U_{k}$, satisfying the following conditions:

(1) $A=A_{1} \cup \cdots \cup A_{k}$;

(2) For each $i \in\{1, \ldots, k\}, A_{i} \subseteq U_{i}$;

(3) For each $i \in\{1, \ldots, k\}$, there exists $j_{i} \in\{1, \ldots, n\}$ such that $U_{i} \subseteq V_{j_{i}}$; and

(4) For each $j \in\{1, \ldots, n\}$, there exists $r_{j} \in\{1, \ldots, k\}$ such that $U_{r_{j}} \subseteq V_{j}$.

Proof. Suppose that $A \in\left\langle V_{1}, \ldots, V_{n}\right\rangle$ satisfies the hypothesis. Then $A$ is a compact zero-dimensional space (it is well-known that every locally compact totally disconnected Hausdorff space is zero-dimensional and completely regular). Then we can find open subsets $W_{1}, \ldots, W_{k}$ of $X$ such that for every $i \leq k$ there is $j \leq n$ such that $\overline{W_{i}} \subseteq U_{j},\left\{A \cap W_{i}: i \leq k\right\}$ is pairwise disjoint and $A \cap W_{i}=A \cap \overline{W_{i}}$ for every $i \leq k$. Thus, we define $U_{i}=W_{i}-\bigcup\left\{\overline{W_{j}}: i \neq j\right\}$ and $A_{i}=A \cap \overline{W_{i}}$ for every $i \leq k$. Clearly $\left\{A_{i}: A_{i} \neq \emptyset\right\}$ and $\left\{U_{i}: U_{i} \neq \emptyset\right\}$ satisfies the Lemma.

Next proposition follows from Lemma 3.1, see also [8, Lemma 2.3.1].

Proposition 3.2. If $X$ is a compact Hausdorff space, then the collection $\left\{\left\langle V_{1}, \ldots, V_{n}\right\rangle: n \in \mathbb{N}\right.$ and $\left.\left\{V_{1}, \ldots, V_{n}\right\} \in \mathfrak{C}(X)\right\}$ is a base for the Vietoris topology on $T D(X)$.

Proposition 3.3. If $X$ is a regular space, then $T D(X)$ has nonempty interior in $2^{X}$ if and only if $X$ contains a nonempty open totally disconnected subset.

Proof. Suppose that $T D(X)$ has nonempty interior in $2^{X}$. Let $U_{1}, \ldots, U_{n}$ be nonempty open subsets of $X$ such that $\left\langle U_{1}, \ldots, U_{n}\right\rangle \subset T D(X)$. For each $i \in\{1, \ldots, n\}$ let $x_{i}$ be a point in $U_{i}$. Let $V$ be an open subset of $X$ such that $x_{1} \in V$ and $\bar{V} \subset U_{1}$. We assert that $V$ is a totally disconnected subset of $X$. Let $A$ be a nonempty connected subset of $V$. Let $B=\bar{A} \cup\left\{x_{1}, \ldots, x_{n}\right\}$. Clearly $B \in\left\langle U_{1}, \ldots, U_{n}\right\rangle$, so $B \in T D(X)$. It follows that $A$ is a one-point set. 
Conversely, if $U$ is a nonempty open totally disconnected subset of $X$, then $\langle U\rangle$ is a nonempty open set contained in $T D(X)$. Thus, $T D(X)$ has nonempty interior in $2^{X}$.

COROLlary 3.4. If $X$ is a compact connected Hausdorff space, then $T D(X)$ has empty interior in $2^{X}$.

In $[8$, Theorem 4.2] it is proved that compactness of a subset of the hyperspace $2^{X}$ containing the singletons of $X$ implies compactness of the space $X$. As a consequence of this fact we have the next proposition. We recall that all the spaces that we consider are $T_{1}$-spaces.

Proposition 3.5. If $X$ is a space such that $T D(X)$ is compact, then $X$ is compact.

Proposition 3.6. If $X$ is a Hausdorff space such that $T D(X)$ is compact, then $X$ is totally disconnected.

Proof. By Proposition 3.5, we have that $X$ is compact. Thus, $X$ is a normal space. Consequently $2^{X}$ is a Hausdorff space, [8, Theorem 4.9, 4.9.3]. Since $T D(X)$ is compact, we have that $T D(X)$ is a closed subset of $2^{X}$. We notice that each finite subset of $X$ belongs to $T D(X)$, so this hyperspace is a dense subset of $2^{X}$ ([8, Proposition 2.4, 2.4.1]). It follows that $T D(X)=2^{X}$. Therefore $X$ is an element of $T D(X)$, which means that $X$ is totally disconnected.

REMARK 3.7. The negation of the last proposition says that if $X$ is a Hausdorff space containing a nondegenerate connected subset, then the hyperspace of totally disconnected subsets of $X$ is not compact. In particular, for each nondegenerate continuum this hyperspace is not compact. In this way we also see that the compactness of $X$ does not imply the compactness of $T D(X)$.

Corollary 3.8. Let $X$ be a Hausdorff space. Then $T D(X)$ is compact if and only if $X$ is compact and totally disconnected.

Proof. Necessity follows from Propositions 3.5 and 3.6. For the converse, we notice that the assumptions imply that $T D(X)$ coincides with $2^{X}$. Therefore, by $[8$, Theorem 4.2], we obtain that $T D(X)$ is compact.

In [8, Theorem 4.10] it is proved that a subset of the hyperspace $2^{X}$ containing every finite set of $X$ is connected if and only if the space $X$ is connected. So, we have next proposition.

Proposition 3.9. For any space $X, T D(X)$ is connected if and only if $X$ is connected. 
In $[8$, Theorem 4.12] it is proved that, for a compact space $X$, a subset of the hyperspace $2^{X}$ containing every finite set of $X$ is locally connected if and only if the space $X$ is locally connected. Similarly, we have the next proposition.

Proposition 3.10. Let $p$ be a point of a space $X$. If $H(X)$ is a subset of $2^{X}$ containing the singletons of $X$ and $H(X)$ is connected im kleinen at $\{p\}$, then $X$ is connected im kleinen at $p$.

Proof. Let $U$ be an open subset of $X$ such that $p \in U$. By hypothesis, there exists a connected subset $\mathcal{V}$ of $H(X)$ such that $\{p\} \in \operatorname{int}_{H(X)}(\mathcal{V})$ and $\mathcal{V} \subseteq\langle U\rangle$. Let $V=\bigcup\{A \in H(X): A \in \mathcal{V}\}$. We have that $V$ is a connected subset of $X$, [10, Lemma (1.43)]. Clearly $p \in V$. We assert that $p \in$ int $_{X}(V)$ and $V \subseteq U$. Let $U_{1}, \ldots, U_{n}$ be open subsets of $X$ such that $\{p\} \in\left\langle U_{1}, \ldots, U_{n}\right\rangle \cap$ $H(X) \subset \operatorname{int}_{H(X)}(\mathcal{V})$ and let $W=U_{1} \cap \cdots \cap U_{n}$. We have that $W$ is an open subset of $X$ containing $p$. If $x$ is a point in $W$ then $x \in U_{i}$ for each $i \in\{1, \ldots, n\}$, so $\{x\} \in\left\langle U_{1}, \ldots, U_{n}\right\rangle \cap H(X) \subseteq \operatorname{int}_{H(X)}(\mathcal{V})$. Thus, $\{x\} \in \mathcal{V}$. Hence, $x \in V$. Thus we have that $W$ is an open subset of $X$ such that $p \in W \subseteq V$. So, $p \in \operatorname{int}_{X}(V)$. Finally, if $x$ is a point of $V$ there exists $A \in \mathcal{V}$ such that $x \in A$. Since $\mathcal{V} \subseteq\langle U\rangle$ we have that $A \subset U$, so $x \in U$. Thus, $V$ is contained in $U$. We have that $V$ is a connected subset of $X$ such that $p \in \operatorname{int}_{X}(V)$ and $V \subseteq U$.

Corollary 3.11. If $X$ is a space such that $T D(X)$ is locally connected, then $X$ is locally connected.

Proposition 3.12. If $X$ is a compact Hausdorff space, then $T D(X)$ is locally connected if and only if $X$ is locally connected.

Proof. Necessity follows from the more general fact stated in Corollary 3.11. Conversely, let $A$ be a nonempty closed totally disconnected subset of $X$ and let $W_{1}, \ldots, W_{n}$ be open subsets of $X$ such that $A$ belongs to $\left\langle W_{1}, \ldots, W_{n}\right\rangle \cap T D(X)$. By Lemma 3.1 there exist $r$ nonempty compact subsets of $A, A_{1}, \ldots, A_{r}$, such that $A=A_{1} \cup \cdots \cup A_{r}, A_{i} \cap A_{j}=\emptyset$ if $i \neq j$ and they satisfy the following conditions.

(1) For each $i \in\{1, \ldots, r\}$, there exists $j_{i} \in\{1, \ldots, n\}$ such that $A_{i} \subseteq W_{j_{i}}$.

(2) For each $j \in\{1, \ldots, n\}$, there exists $r_{j} \in\{1, \ldots, r\}$ such that $A_{r_{j}} \subseteq$ $W_{j}$.

Let $U_{1}, \ldots, U_{r}$ be pairwise disjoint open subsets of $X$ such that $A_{i} \subseteq U_{i}$, for each $i \in\{1, \ldots, r\}$ and we define $V_{i}=U_{i} \cap\left(\cap\left\{W_{j}: A_{i} \subseteq W_{j}\right\}\right)$, for each $i \in\{1, \ldots, r\}$. If $i \in\{1, \ldots, r\}$, for each $x \in A_{i}$ there exists $C_{x}$ an open connected subset of $X$ such that $x \in C_{x}$ and $C_{x} \subseteq V_{i}$. Since $A_{i}$ is compact there exists a finite subset, $\left\{x_{i 1}, \ldots, x_{i k_{i}}\right\}$, such that $A_{i} \subseteq C_{x_{i 1}} \cup \cdots \cup C_{x_{i k_{i}}}$. Let $D_{i}=C_{x_{i 1}} \cup \cdots \cup C_{x_{i k_{i}}}$. Observe that $D_{i}$ has only a finite number of 
components, $M_{1}^{i}, \ldots, M_{p_{i}}^{i}$, and every $M_{j}^{i}$ is an open subset of $X$. Let

$$
\mathcal{V}=\left\langle M_{1}^{1}, \ldots, M_{p_{1}}^{1}, \ldots, M_{1}^{r}, \ldots, M_{p_{r}}^{r}\right\rangle \cap T D(X) .
$$

Clearly $\mathcal{V}$ is an open subset of $T D(X)$. We assert that $\mathcal{V}$ is connected. We know that $\mathcal{V} \cap F_{m}(X)$ is connected, for each $m \geq p_{1}+\cdots+p_{r},[6$, Lemma 1], where $F_{m}(X)$ denotes the hyperspace of all nonempty subsets of $X$ having at most $m$ points. Let $\mathcal{U}=\cup\left\{\mathcal{V} \cap F_{m}(X): m \geq p_{1}+\cdots+p_{r}\right\}$, we have that $\mathcal{U}$ is a connected subset of $T D(X)$. Now, since $\cup\left\{F_{m}(X): m \geq p_{1}+\cdots+p_{r}\right\}$ is a dense subset of $T D(X)$ and $\mathcal{U}=\mathcal{V} \cap\left(\cup\left\{F_{m}(X): m \geq p_{1}+\cdots+p_{r}\right\}\right)$, we have that $\mathcal{U}$ is dense in $\mathcal{V}$. Thus, $\mathcal{V}$ is connected. We will see that $A \in \mathcal{V}$. Observe that $A=\bigcup_{i=1}^{r} A_{i} \subseteq \bigcup_{i=1}^{r}\left(\bigcup_{j=1}^{k_{i}} C_{x_{i j}}\right)=\bigcup_{i=1}^{r}\left(\bigcup_{j=1}^{p_{i}} M_{j}^{i}\right)$. For each $M_{j}^{i}$, there exists a point $x_{i l} \in\left\{x_{i 1}, \ldots, x_{i k_{i}}\right\} \subseteq A_{i}$ such that $C_{x_{i l}} \subseteq M_{j}^{i}$, so $A_{i} \cap M_{j}^{i} \neq \emptyset$, thus $A \cap M_{j}^{i} \neq \emptyset$. This shows that $A \in \mathcal{V}$. Finally we assert that $\mathcal{V} \subseteq$ $\left\langle W_{1}, \ldots, W_{n}\right\rangle$. Let $B \in \mathcal{V}$. According to condition (2), if $j \in\{1, \ldots, n\}$, there exists $r_{j} \in\{1, \ldots, r\}$ such that $A_{r_{j}} \subseteq W_{j}$. Since $B \cap\left(\bigcup_{l=1}^{p_{r_{j}}} M_{l}^{r_{j}}\right) \neq \emptyset$ and $B \cap\left(\bigcup_{l=1}^{p_{r_{j}}} M_{l}^{r_{j}}\right) \subseteq W_{j}$ we have that $B \cap W_{j} \neq \emptyset$, so $B \cap W_{j} \neq \emptyset$, for each $j \in\{1, \ldots, n\}$. Now, if $i \in\{1, \ldots, r\}$ and $l \in\left\{1, \ldots, p_{i}\right\}$ then $M_{l}^{i}$ is a component of $D_{i}$ and by definition of $D_{i}$ we have that $D_{i} \subseteq V_{i}$ and $V_{i} \subseteq W_{j_{i}}$, for some $j_{i} \in\{1, \ldots, n\}$. Therefore $B \in\left\langle W_{1}, \ldots, W_{n}\right\rangle$. We have shown that $\mathcal{V}$ is an open connected subset of $T D(X)$ such that $A \in \mathcal{V} \subseteq\left\langle W_{1}, \ldots, W_{n}\right\rangle$.

\section{Path CONnECTEDness}

In this section, we prove that for every smooth dendroid $X$ the hyperspace $T D(X)$ is contractible, so it is pathwise connected, and we include an example of a (non-smooth) dendroid for which this hyperspace is not pathwise connected. We also prove that for a Hausdorff, locally compact, not compact and Lindelöf space $X$, the hyperspace $T D(X)$ has uncountably many arc components.

THEOREM 4.1. If $X$ is a smooth dendroid, then $T D(X)$ is contractible.

Proof. Let $p$ be a point of smoothness of $X$. By [9, Theorem 1.16] there is a continuous function, $H: X \times[0,1] \rightarrow X$, such that for each $x \in X$ and for each $t \in[0,1], H$ satisfies the following conditions:

(i) $H((x, 0))=p$;

(ii) $d(H((x, t)), p)=t$, if $t \leq d(x, p)$; and

(iii) $H((x, t))=x$, if $d(x, p) \leq t$.

We define $\mathcal{H}: T D(X) \times[0,1] \rightarrow 2^{X}$ by $\mathcal{H}((A, t))=H(A \times\{t\})$ for each $(A, t) \in T D(X) \times[0,1]$. We will prove that $\mathcal{H}$ is the required homotopy. Let $B_{t}=\{x \in X: d(x, p)=t\}$ and $F_{t}=\{x \in X: d(x, p) \leq t\}$ for each $t$ in 
the open interval $(0,1)$. Notice that for each $x \in X, H((x, t)) \in F_{t}$. We will prove that each $B_{t}$ is a totally disconnected set. Fix $t \in(0,1)$. Let $x \in B_{t}$ and $K$ be the component of $x$ in $B_{t}$. Suppose that there exists $z \in K-\{x\}$. Thus $K$ is a nondegenerate subdendroid of $X$. We have that $x z \subset K$. Since $t>0$, we have that $p$ is not in $B_{t}$, thus $p$ is not in $x z$. Let $w$ be the first time that the arc $p x$ intersects the arc $x z$. Thus $w \in x z$ and $p w \cap x z=\{w\}$. Notice that $p \neq w$. Without loss of generality, assume that $z \neq w$. The fact that $p, z \in H(\{z\} \times[0, t])\left(z \in F_{t}\right)$ and $H(\{z\} \times[0, t])$ is connected implies that $w \in H(\{z\} \times[0, t])(p z=p w \cup w z)$. There exists $r \in[0, t]$ such that $H((z, r))=w$. If $r=t$, then $w=H((z, t))=z\left(z \in B_{t} \subseteq F_{t}\right)$ and hence $w=z$ but this is a contradiction. Therefore $r \in[0, t)$ and $H((z, r))=w$, i.e. $w \in F_{r}$ with $r<t$, this implies that $w \notin B_{t}$ but this contradicts the fact that $w \in x z \subseteq K \subseteq B_{t}$. Hence, we have proved that $B_{t}$ is totally disconnected.

On the other hand, it is easy to see that $\mathcal{H}$ is a continuous function. Now by conditions $(i)$ and $($ iii $)$ we have that $\mathcal{H}((A, 0))=\{p\}$ and $\mathcal{H}((A, 1))=A$ for each $A \in T D(X)$ (we are assuming that $\operatorname{maximum}\{d(x, y): x, y \in X\}=1$ ). We will prove that $\mathcal{H}((A, t)) \in T D(X)$ for each $t \in(0,1)$ and each $A \in$ $T D(X)$. Let $t \in(0,1)$ and $A \in T D(X)$. Since for each $x \in X, H((x, t)) \in F_{t}$, we have that $H(A \times\{t\}) \subseteq F_{t}$. Hence according to conditions (ii) and (iii), $H(A \times\{t\}) \subseteq\left(A \cap F_{t}\right) \cup B_{t}$. In order to prove that $\mathcal{H}((A, t))$ is a totally disconnected set, we will prove that $\left(A \cap F_{t}\right) \cup B_{t}$ is totally disconnected. Let $C$ be a component of $\left(A \cap F_{t}\right) \cup B_{t}$. Suppose that $|C| \geq 2$. Since $B_{t}$ is totally disconnected we have that $C \cap\left(\left(A \cap F_{t}\right)-B_{t}\right) \neq \emptyset$. Let $a \in C \cap\left(A \cap F_{t}-B_{t}\right)$. By $\left[10,(1.8)\right.$ and (1.11)], there is an order arc, $\gamma:[0,1] \rightarrow\left\{E \in 2^{X}\right.$ : $E$ is connected $\}$, from $\{a\}$ to $C$. Since $\gamma(0)=\{a\} \in\left\langle X-B_{t}\right\rangle$ there is $r>0$ such that $\gamma(r) \in\left\langle X-B_{t}\right\rangle$. We have that $\gamma(r) \subseteq A \cap F_{t}$ and $\gamma(r)$ is a nondegenerate connected subset of $A \cap F_{t}$, this contradicts that $A \cap F_{t}$ is totally disconnected. Thus $|C| \leq 1$. Hence, we have proved that $\mathcal{H}((A, t))$ is totally disconnected. Therefore $T D(X)$ is contractible.

Corollary 4.2. If $X$ is a smooth dendroid, then $T D(X)$ is pathwise connected.

Corollary 4.3. If $X$ is a dendrite, then $T D(X)$ is pathwise connected.

Problem 4.1. Is it true that if $X$ is a contractible dendroid, then $T D(X)$ is contractible?

Problem 4.2. Give conditions on $X$ under which $T D(X)$ is contractible.

Next we give an example of a (non-smooth) dendroid $X$ whose hyperspace $T D(X)$ is not pathwise connected. In order to do this we include the following lemma that can be proved in the same way as Lemma 2.7 of [3].

Lemma 4.4. Let $X$ be a regular space. If $f:[0,1] \rightarrow T D(X)$ is a continuous function, $C$ is a nonempty closed subset of $\bigcup f([0,1])$ and $u=\inf \{s \in[0,1]: f(s) \cap C \neq \emptyset\}$, then $f(u) \cap C \neq \emptyset$. 
Also we recall that a continuum $X$ is uniformly arcwise connected if it is arcwise connected and for each number $\varepsilon>0$ there is a positive integer $k$ such that every arc $A$ contained in $X$ contains points $a_{0}, a_{1}, \ldots, a_{k}$ such that $A=a_{0} a_{1} \cup \cdots \cup a_{k-1} a_{k}$ and $\operatorname{diam}\left(a_{i} a_{i+1}\right)<\varepsilon$ for each $i \in\{0, \ldots, k-1\},[1$, D5].

EXAmple 4.5. A dendroid $X$ such that $T D(X)$ is not pathwise connected.

Let $L_{0}$ be the line segment in the plain from the point $(0,0)$ to the point $(1,0)$. For each $n \in \mathbb{N}$, let $x_{n}=\left(1, \frac{1}{n}\right), y_{i n}=\left(1, \frac{1}{n}-\frac{i}{2 n^{2}(n+1)}\right)$ with $i \in$ $\{1, \ldots, n\}$ and $z_{i n}=\left(\frac{3}{4}, \frac{3}{4}-\left(\frac{3}{4}\right) \frac{i}{2 n^{2}(n+1)}\right)$ for each $i \in\{1, \ldots, n\}$. For each $n \in \mathbb{N}$ let $H_{n}$ be the line segment of the point $x_{n}$ to the point $z_{1 n}, I_{n}$ the line segment of the point $(0,0)$ to the point $x_{n}, J_{i n}$ the line segment of the point $z_{i n}$ to the point $y_{i n}$ for each $i \in\{1, \ldots, n\}$ and $K_{i n}$ the line segment of the point $y_{\text {in }}$ to the point $z_{(i+1) n}$ for each $i \in\{1, \ldots, n-1\}$. For each $n \in \mathbb{N}$, let $L_{n}=H_{n} \cup I_{n} \cup\left(\bigcup\left\{J_{\text {in }}: i \in\{1, \ldots, n\}\right\}\right) \cup\left(\bigcup\left\{K_{\text {in }}: i \in\{1, \ldots, n-1\}\right\}\right)$ and $X=L_{0} \cup\left(\bigcup\left\{L_{n}: n \in \mathbb{N}\right\}\right)$, see Figure 1. We will prove that $T D(X)$ is not pathwise connected.

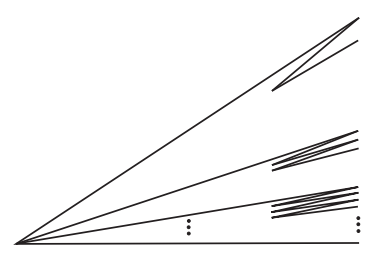

Figure 1. A dendroid with non-pathwise connected hyperspace $T D(X)$

Let $S_{0}=\{(0,0)\}$ and $S_{1}=\left\{x_{n}: n \in \mathbb{N}\right\} \bigcup\{(1,0)\} \bigcup\left\{y_{\text {in }}: n \in \mathbb{N}\right.$ and $i \in$ $\{1, \ldots, n\}\}$. Suppose there is a path $f:[0,1] \rightarrow T D(X)$ such that $f(0)=$ $S_{0}$ and $f(1)=S_{1}$. We notice that $S_{0}$ is contained in a uniformly arcwise connected subcontinuum of $X$ and $S_{1}$ is not. Let $r$ be the least upper bound of the set of all numbers $s \in[0,1]$ such that $f(t)$ is contained in a uniformly arcwise connected subcontinuum of $X$ for each $t \leq s$. By Proposition 3.2 we can choose pairwise disjoint open sets $U_{1}, \ldots, U_{k}$ of $X$ such that $\operatorname{diam}\left(U_{i}\right)<\frac{1}{8}$, for each $i \in\{1, \ldots, k\}$ and $f(r) \in \mathcal{U}=\left\langle U_{1}, \ldots, U_{k}\right\rangle$. We have to consider two cases:

CASE 1. $f(r)$ is contained in a uniformly arcwise connected subcontinuum of $X$. Clearly $0 \leq r<1$. Let $t_{0} \in(r, 1]$ such that $f\left(\left[r, t_{0}\right]\right) \subseteq \mathcal{U}$ and $f\left(t_{0}\right)$ is not contained in a uniformly arcwise connected subcontinuum of $X$. We have that for each $n \in \mathbb{N}$ there exists $N \in \mathbb{N}$ with $N>n$ such that $f\left(t_{0}\right) \cap J_{i N} \neq \emptyset$ or $f\left(t_{0}\right) \cap K_{i N} \neq \emptyset$ for some $i>N$. Then $f\left(t_{0}\right)$ has a limit point in the line segment from the point $\left(\frac{3}{4}, 0\right)$ to the point $(1,0)$. Let $x_{0}$ be a point 
limit of $f\left(t_{0}\right)$ in the line segment from the point $\left(\frac{3}{4}, 0\right)$ to the point $(1,0)$. Without loss of generality assume that $x_{0} \in U_{1}$. Since $f(r)$ is contained in a uniformly arcwise connected subcontinuum of $X$ there exists $m \in \mathbb{N}$ such that $f(r) \cap J_{i n}=\emptyset$ for each $n \geq m$ and for each $i \in\{m, \ldots, n\}$; and $f(r) \cap K_{i n}=\emptyset$ for each $n \geq m$ and for each $i \in\{m, \ldots, n-1\}$. Now choose a connected component $C$ of $U_{1}$ such that $f\left(t_{0}\right) \cap C \cap\left(J_{j N} \cup K_{l M}\right) \neq \emptyset$ with $N, M>2 m$, $j \in\{2 m, \ldots, N\}$ and $l \in\{2 m, \ldots, M\}$. We notice that $f(r) \cap C=\emptyset$. Let $D=C \cap\left[\bigcup f\left(\left[r, t_{0}\right]\right)\right]$. Observe that $D$ is a closed subset of $\bigcup f\left(\left[r, t_{0}\right]\right)$. Since $f_{\left.\mid r, t_{0}\right]}$ and $D$ satisfy the conditions of Lemma $4.4, f(u) \cap D \neq \emptyset$ where $u=\inf \left\{s \in\left[r, t_{0}\right]: f(s) \cap D \neq \emptyset\right\}$. Since $f(r) \cap C=\emptyset$ and $D \subseteq C$ we have that $r<u$. Consider the open sets $C$ and $\mathcal{V}=\left\langle C, U_{1}, \ldots, U_{m}\right\rangle$. Since $f(u) \cap D \neq \emptyset$ and $f(u) \in f\left(\left[r, t_{0}\right]\right) \subseteq \mathcal{U}$ we have that $f(u) \in \mathcal{V}$. Then $f^{-1}(\mathcal{V})$ is an open subset of $[0,1]$ such that $u \in f^{-1}(\mathcal{V})$ and $f^{-1}(\mathcal{V}) \cap[r, u)=\emptyset$, which contradicts the continuity of $f$.

CASE 2. $f(r)$ is not contained in a uniformly arcwise connected subcontinuum of $X$. In this case, we notice that $0<r$ and for each $n \in \mathbb{N}$ there exists $N \in \mathbb{N}$ with $N>n$ such that either $f(r) \cap J_{i N} \neq \emptyset$ or $f(r) \cap K_{i N} \neq \emptyset$ for some $i>N$. We have that $f(r)$ has a limit point in the line segment from the point $\left(\frac{3}{4}, 0\right)$ to the point $(1,0)$. Let $x_{0}$ be a limit point of $f(r)$ in the line segment from the point $\left(\frac{3}{4}, 0\right)$ to the point $(1,0)$. Without loss of generality assume that $x_{0} \in U_{1}$. Let $t_{0}$ be a point in $[0, r)$ such that $f\left(\left[t_{0}, r\right]\right) \subseteq \mathcal{U}$. Observe that $f\left(t_{0}\right)$ is contained in a uniformly arcwise connected subcontinuum of $X$. Then, we may take $m \in \mathbb{N}$ such that $f\left(t_{0}\right) \cap J_{\text {in }}=\emptyset$ for each $n \geq m$ and for each $i \in\{m, \ldots, n\}$; and $f\left(t_{0}\right) \cap K_{i n}=\emptyset$ for each $n \geq m$ and for each $i \in\{m, \ldots, n-1\}$. We choose a connected component $C$ of $U_{1}$ such that $f(r) \cap C \cap\left(J_{j N} \cup K_{l M}\right) \neq \emptyset$ with $N, M>2 m$, $j \in\{2 m, \ldots, N\}$ and $l \in\{2 m, \ldots, M\}$. We have that $f\left(t_{0}\right) \cap C=\emptyset$. Let $D=C \cap\left[\bigcup f\left(\left[t_{0}, r\right]\right)\right]$. Observe that $D$ is a closed subset of $\bigcup f\left(\left[t_{0}, r\right]\right)$. By Lemma 4.4, if $u=\inf \left\{s \in\left[t_{0}, r\right]: f(s) \cap D \neq \emptyset\right\}$, then $f(u) \cap D \neq \emptyset$. Since $f\left(t_{0}\right) \cap C=\emptyset$ and $D \subseteq C$ we have that $t_{0}<u$. Consider the open sets $C$ and $\mathcal{V}=\left\langle C, U_{1}, \ldots, U_{m}\right\rangle$. Since $f(u) \cap D \neq \emptyset$ and $f(u) \in f\left(\left[t_{0}, r\right]\right) \subseteq \mathcal{U}$ we have that $f(u) \in \mathcal{V}$. Then $f^{-1}(\mathcal{V})$ is an open subset of $[0,1]$ such that $u \in f^{-1}(\mathcal{V})$ and $f^{-1}(\mathcal{V}) \cap\left[t_{0}, u\right)=\emptyset$, but this also contradicts the continuity of $f$. Therefore $S_{0}$ and $S_{1}$ cannot be connected by a path in $T D(X)$. This proves that for the dendroid in Figure 1 the hyperspace of totally disconnected sets is not pathwise connected.

Note that the dendroid in the previous example is not uniformly arcwise connected and that smooth dendroids are uniformly arcwise connected, see [2, Corollary 16]. In this context it is interesting to ask:

Problem 4.3. Is TD $(X)$ pathwise connected for every uniformly arcwise connected continuum $X$ ? 
Problem 4.4. Let $X$ be a continuum that is not uniformly arcwise connected. Is it true that $T D(X)$ has $\mathfrak{c}$ arc components?

Next we will present a class of spaces, that include the Euclidean spaces $\mathbb{R}^{n}$, for which the hyperspace of totally disconnected subsets has at least $\mathfrak{c}$ path components, see Theorem 4.11 below. First we state several technical lemmas.

LEMma 4.6. Let $X$ be a normal space such that $\operatorname{dim}(X)=0$. If $Y=\left\{y_{n}\right.$ : $n \in \mathbb{N}\}$ is a discrete, infinite and closed subset of $X$ and $\left\{Q_{n}: n \in \mathbb{N}\right\}$ is a cellular family of closed-open subsets of $X$ such that $y_{n} \in Q_{n}$ for each $n \in \mathbb{N}$, then there exists a cellular family of closed-open subsets of $X,\left\{V_{n}: n \in \mathbb{N}\right\}$, such that $y_{n} \in V_{n}$ for each $n \in \mathbb{N}$ and $\bigcup\left\{V_{n}: n \in \mathbb{N}\right\} \in 2^{X}$. Moreover, if $\left\{P_{n}: n \in \mathbb{N}\right\} \subseteq 2^{X}$ satisfies that $P_{n} \subseteq V_{n}$ for each $n \in \mathbb{N}$, then $\bigcup\left\{P_{n}: n \in\right.$ $\mathbb{N}\} \in 2^{X}$.

Proof. Let $L=\overline{\bigcup\left\{Q_{n}: n \in \mathbb{N}\right\}}-\bigcup\left\{Q_{n}: n \in \mathbb{N}\right\}$. If $L=\emptyset$, then the Lemma is true. Suppose that $L$ is not empty. Since $X$ is a normal space, there exists an open subset of $X, W_{0}$ such that $Y \subseteq W_{0} \subseteq \overline{W_{0}} \subseteq X-L$. Observe that for each $n \in \mathbb{N}, y_{n} \in Q_{n} \cap W_{0}$ and $Q_{n} \cap W_{0}$ is an open subset of $X$. Since $\operatorname{dim}(X)=0$, for each $n \in \mathbb{N}$, there exists a closed-open subset of $X, V_{n}$, such that $y_{n} \in V_{n} \subseteq Q_{n} \cap W_{0}$. Clearly $\left\{V_{n}: n \in \mathbb{N}\right\}$ is a cellular family in $X$. We assert that $\bigcup\left\{V_{n}: n \in \mathbb{N}\right\} \in 2^{X}$. Since $V_{n} \subseteq Q_{n} \cap W_{0}$, for each $n \in \mathbb{N}$, we have that $\bigcup\left\{V_{n}: n \in \mathbb{N}\right\} \subseteq\left(\bigcup\left\{Q_{n}: n \in \mathbb{N}\right\}\right) \cap W_{0}$, consequently $\overline{\bigcup\left\{V_{n}: n \in \mathbb{N}\right\}} \subseteq$ $\overline{\bigcup\left\{Q_{n}: n \in \mathbb{N}\right\}} \cap \overline{W_{0}} \subseteq \overline{\bigcup\left\{Q_{n}: n \in \mathbb{N}\right\}} \cap(X-L)$. So $\overline{\bigcup\left\{V_{n}: n \in \mathbb{N}\right\}} \subseteq \bigcup\left\{Q_{n}:\right.$ $n \in \mathbb{N}\}$. Now, we take a point $x$ in $\overline{\bigcup\left\{V_{n}: n \in \mathbb{N}\right\}}$. We have that $x \in Q_{n}$, for some $n \in \mathbb{N}$. If $x \in Q_{n}-V_{n}$, then $\left(Q_{n}-V_{n}\right) \cap\left(\bigcup\left\{V_{n}: n \in \mathbb{N}\right\}\right)=\emptyset$ $\left(Q_{n} \cap Q_{m}=\emptyset\right.$, if $\left.n \neq m\right)$, which is a contradiction. Therefore, $x \in V_{n}$. This proves that $\bigcup\left\{V_{n}: n \in \mathbb{N}\right\} \in 2^{X}$. Similarly one can prove that if $\left\{P_{n}: n \in\right.$ $\mathbb{N}\} \subseteq 2^{X}$ satisfies that $P_{n} \subseteq V_{n}$ for each $n \in \mathbb{N}$, then $\bigcup\left\{P_{n}: n \in \mathbb{N}\right\} \in 2^{X}$.

Lemma 4.7. Let $X$ be a normal space. If $\left\{P_{n}: n \in \mathbb{N}\right\}$ is a family of subsets of $X$ such that $P_{n} \cap P_{m}=\emptyset$ if $n \neq m, \bigcup\left\{P_{n}: n \in \mathbb{N}\right\} \in 2^{X}$ and $P_{n}$ is closed-open subset of $\bigcup\left\{P_{n}: n \in \mathbb{N}\right\}$ for each $n \in \mathbb{N}$, then there exists a cellular family, $\left\{U_{n}: n \in \mathbb{N}\right\}$, such that $P_{n} \subseteq U_{n}$ for each $n \in \mathbb{N}$.

Proof. Observe that for each $m \in \mathbb{N}, P_{m}$ and $\bigcup\left\{P_{n}: n \in \mathbb{N}-\{m\}\right\}$ are closed subsets of $\bigcup\left\{P_{n}: n \in \mathbb{N}\right\}$, so $P_{m}$ and $\bigcup\left\{P_{n}: n \in \mathbb{N}-\{m\}\right\}$ are closed disjoint subsets of $X$. Since $X$ is a normal space, for each $m \in \mathbb{N}$, there exists an open subset of $X, V_{m}$ such that $P_{m} \subseteq V_{m}$ and $\overline{V_{m}} \cap \bigcup\left\{P_{n}\right.$ : $n \in \mathbb{N}-\{m\}\}=\emptyset$. Let $U_{1}=V_{1}$ and $U_{m}=V_{m}-\left(\overline{V_{1}} \cup \cdots \cup \overline{V_{m-1}}\right)$. Clearly $\left\{U_{m}: m \in \mathbb{N}\right\}$ satisfies the Lemma.

LEMMA 4.8. Let $X$ be a locally compact and normal space and let $E, B \in$ $T D(X)$. If $Y$ is a discrete, countably infinite and closed subset of $\overline{E-B}$, 
then there exists an open subset of $X, V$, and for each $n \in \mathbb{N}$ there exists an open subset of $X, U_{n}$, satisfying the following conditions:

(1) For each $n \in \mathbb{N}$ we have that $V \cap U_{n}=\emptyset$ and $U_{n} \cap U_{m}=\emptyset$, if $n \neq m$.

(2) $B \subseteq V$; and for each $n \in \mathbb{N}, U_{n} \cap E \neq \emptyset$.

(3) $E \subseteq V \cup\left(\bigcup\left\{U_{n}: n \in \mathbb{N}\right\}\right)$.

Proof. Let $Y=\left\{y_{n}: n \in \mathbb{N}\right\}$. We know that for each $n \in \mathbb{N}$ there exists an open subset of $X, W_{n}$, such that $y_{n} \in W_{n}$ and $W_{n} \cap W_{m}=\emptyset$ if $n \neq m$. We also know that $E$ has dimension zero, consequently, for each $n \in \mathbb{N}$ there exists a closed-open subset of $E, O_{n}$, such that $y_{n} \in O_{n} \subseteq W_{n}$. Now, by Lemma 4.6, there exists a cellular family of closed-open subsets of $E,\left\{V_{n}: n \in \mathbb{N}\right\}$, such that $y_{n} \in V_{n}$ for each $n \in \mathbb{N}$ and $\bigcup\left\{V_{n}: n \in \mathbb{N}\right\} \in 2^{E}$. Observe that $\bigcup\left\{V_{n}: n \in \mathbb{N}\right\} \in 2^{X}$. Since for each $n \in \mathbb{N}, y_{n} \in \overline{E-B}$ and $y_{n} \in V_{n}$ there exists a point $x_{n}$ in $V_{n} \cap(E-B)=V_{n}-B$. Since $\operatorname{dim}(E)=0$ we have that for each $n \in \mathbb{N}$ there exists a closed-open subset of $E, P_{n}$, such that $x_{n} \in P_{n} \subseteq V_{n}-B$. Now, by Lemma 4.6 we may assume $\bigcup\left\{P_{n}: n \in \mathbb{N}\right\} \in 2^{E}$ so $\bigcup\left\{P_{n}: n \in \mathbb{N}\right\} \in 2^{X}$. Observe that $\left\{P_{n}: n \in \mathbb{N}\right\}$ satisfies the assumptions of the Lemma 4.7, consequently for each $n \in \mathbb{N}$ there exists an open subset of $X, Q_{n}$, such that $P_{n} \subseteq Q_{n}$ and $Q_{n} \cap Q_{m}=\emptyset$ if $n \neq m$. On the other hand, observe that $\bigcup\left\{P_{n}: n \in \mathbb{N}\right\} \subseteq E-B$. Thus $B \cup\left(E-\bigcup\left\{P_{n}: n \in \mathbb{N}\right\}\right)$ and $\bigcup\left\{P_{n}: n \in \mathbb{N}\right\}$ are disjoint closed subsets of $X$. Since $X$ is a normal space there exist disjoint open subsets of $X, V$ and $W$, such that $B \cup\left(E-\bigcup\left\{P_{n}: n \in \mathbb{N}\right\}\right) \subseteq V$ and $\bigcup\left\{P_{n}: n \in \mathbb{N}\right\} \subseteq W$. For each $n \in \mathbb{N}$ let $U_{n}=Q_{n} \cap W$. Clearly $\{V\} \cup\left\{U_{n}: n \in \mathbb{N}\right\}$ satisfies conditions (1) and (3). Finally in order to see condition (2), observe that for each $n \in \mathbb{N}$, $x_{n} \in P_{n} \subseteq Q_{n} \cap W \cap E=U_{n} \cap E$.

Lemma 4.9. Let $X$ be a space and let $\mathcal{A}$ be a connected subset of $2^{X}$. If $K$ is a nonempty open and closed subset of $\bigcup \mathcal{A}$, then $A \cap K \neq \emptyset$ for each $A \in \mathcal{A}$.

Proof. Assume that there exists $A \in \mathcal{A}$ such that $A \cap K=\emptyset$. Since $K$ is an open subset of $\cup \mathcal{A}$, there exists an open subset $U$ of $X$ such that $K=U \cap(\bigcup \mathcal{A})$. We have that $\langle K, X\rangle \cap \mathcal{A}=\langle U, X\rangle \cap \mathcal{A}$, which is an open subset of $\mathcal{A}$. Now, since $K$ is a closed subset of $\bigcup \mathcal{A}$ we have that $(\bigcup \mathcal{A})-K$ is an open subset of $\cup \mathcal{A}$, consequently there exists an open subset $V$ of $X$ such that $(\bigcup \mathcal{A})-K=V \cap(\bigcup \mathcal{A})$. We have that $\langle(\bigcup \mathcal{A})-K\rangle \cap \mathcal{A}=\langle V\rangle \cap \mathcal{A}$, which is an open subset of $\mathcal{A}$. Note that $\mathcal{A}=[\langle(\cup \mathcal{A})-K\rangle \cap \mathcal{A}] \cup[\langle K, X\rangle \cap \mathcal{A}]$ and that $A \in\langle\bigcup(\mathcal{A})-K\rangle \cap \mathcal{A}$. Also, since $K \neq \emptyset$ and $K \subseteq \bigcup \mathcal{A}$ we have that $\langle K, X\rangle \cap \mathcal{A} \neq \emptyset$. Observe that $[\langle(\cup \mathcal{A})-K\rangle \cap \mathcal{A}] \cap[\langle K, X\rangle \cap \mathcal{A}]=\emptyset$. Therefore $\mathcal{A}$ is not connected.

Lemma 4.10. Let $X$ be a locally compact and Hausdorff space. Let $K$ be a compact subset of $X, P \in 2^{X}$ and $B \subseteq X$ such that $P-K \subseteq B$. Suppose that $P-K=\left\{c_{n}: n \in \mathbb{N}\right\} \in 2^{X}$ is discrete and $c_{n} \neq c_{m}$ for each $n \neq m$. If 
there exists $\left\{P_{k}: k \in \mathbb{N}\right\} \subseteq 2^{X}$ such that $P_{k} \rightarrow P$ and $\overline{P_{k}-B}$ is not compact for each $k \in \mathbb{N}$, then there exist an open set $V, M \in \mathbb{N}$, and for each $m \in \mathbb{N}$, an open set $U_{m}$ and $n_{m} \in \mathbb{N}$ such that:

(1) The elements of $\left\{U_{m}: m \in \mathbb{N}\right\} \cup\{V\}$ are pairwise disjoint.

(2) $K \subseteq V$ and $c_{m} \in U_{m}$, for each $m \in \mathbb{N}$.

(3) $n_{m}<n_{m+1}$, for each $m \in \mathbb{N}$.

(4) For each $m \in \mathbb{N}$, there exists $x_{m} \in P_{M+m} \cap\left(U_{n_{m}}-\left\{c_{n_{m}}\right\}\right)$.

Proof. There exists an open subset $V$ of $X$ such that $K \subseteq V \subseteq \bar{V} \subseteq$ $X-(P-K)$ and $\bar{V}$ is compact. We know that for each $m \in \mathbb{N}$, there exists an open subset $V_{m}$ of $X$ such that $c_{m} \in V_{m}$ and $V_{n} \cap V_{m}=\emptyset$, if $n \neq m$. Now, for each $m \in \mathbb{N}$, there exist open subsets $W_{m}$ and $W_{m}^{\prime}$ of $X$ such that $c_{m} \in W_{m}, \bar{V} \subseteq W_{m}^{\prime}$ and $W_{m} \cap W_{m}^{\prime}=\emptyset$. For each $m \in \mathbb{N}$, we define $U_{m}^{\prime}=W_{m} \cap V_{m}$. For each $m \in \mathbb{N}$ there exists an open subset $U_{m}$ of $X$ such that $c_{m} \in U_{m} \subseteq \overline{U_{m}} \subseteq U_{m}^{\prime}$ and $\overline{U_{m}}$ is compact. It is easy to verify that $\left\{U_{m}: m \in \mathbb{N}\right\} \cup\{V\}$ satisfies conditions (1) and (2). In order to prove (3) and (4) let $U=V \cup\left(\bigcup\left\{U_{m}: m \in \mathbb{N}\right\}\right)$. Observe that $P \in\langle U\rangle$. Since $P_{k} \rightarrow P$, there exists $M \in \mathbb{N}$ such that $P_{k} \in\langle U\rangle$, for each $k>M$. Suppose that $\overline{P_{M+1}-B} \subseteq \bar{V}$. Since $\bar{V}$ is compact, we have that $\overline{P_{M+1}-B}$ is compact, which is a contradiction. Then, there is $y_{1} \in \overline{P_{M+1}-B}-\bar{V} \subseteq P_{M+1}-V$. Let $n_{1} \in \mathbb{N}$ such that $y_{1} \in U_{n_{1}}$. Hence, there is $x_{1} \in\left(P_{M+1}-B\right) \cap U_{n_{1}}$. Observe that $x_{1} \neq c_{n_{1}}$ because $c_{n_{1}} \in P-K \subseteq B$, thus $x_{1} \in\left(P_{M+1}-B\right) \cap$ $\left(U_{n_{1}}-\left\{c_{n_{1}}\right\}\right) \subseteq P_{M+1} \cap\left(U_{n_{1}}-\left\{c_{n_{1}}\right\}\right)$. Suppose that there are $n_{1}<\cdots<n_{j}$ and points $x_{1}, \ldots, x_{j} \in X$ such that $x_{i} \in P_{M+i} \cap\left(U_{n_{i}}-\left\{c_{n_{i}}\right\}\right)$ for each $i \in\{1, \ldots, j\}$. Then, since $\overline{P_{M+j+1}-B} \subseteq P_{M+j+1}$ is not compact and $\bar{V} \cup \overline{U_{n_{1}}} \cup \cdots \cup \overline{U_{n_{j}}}$ is compact, there exists $y_{j+1} \in \overline{P_{M+j+1}-B}-(\bar{V} \cup$ $\left.\overline{U_{n_{1}}} \cup \cdots \cup \overline{U_{n_{j}}}\right)$. Then, there is $n_{j+1} \in \mathbb{N}$ such that $y_{j+1} \in U_{n_{j+1}}$. Let $x_{j+1} \in\left(P_{M+j+1}-B\right) \cap\left(U_{n_{j+1}}-\left\{c_{n_{j+1}}\right\}\right) \subseteq P_{M+j+1} \cap\left(U_{n_{j+1}}-\left\{c_{n_{j+1}}\right\}\right)$.

TheOrem 4.11. Let $X$ be a locally compact, Hausdorff and Lindelöf space. If $C$ is a closed, discrete and countably infinite subset of $X$, and $A, B \in$ $T D(X)$ are subsets of $C$ such that $A-B$ is infinite, then there is no path from $A$ to $B$ in $T D(X)$.

Proof. Suppose that there exists a path in $T D(X)$ such that $\alpha(0)=B$ and $\alpha(1)=A$. Let $T=\{t \in[0,1]: \overline{\alpha(t)-B}$ is compact $\}$ and $t_{0}=\sup T$. Observe that $0 \in T$ and $1 \notin T$. Suppose first that $t_{0} \notin T$, i.e. $\overline{\alpha\left(t_{0}\right)-B}$ is not compact, so it is not countably compact. Then there exists a subset $Y$ of $\overline{\alpha\left(t_{0}\right)-B}$ such that $Y$ is closed, discrete and countably infinite. By Lemma 4.8 there exists an open subset, $V$, of $X$ and for each $n \in \mathbb{N}$ there exists an open subset, $U_{n}$, of $X$ such that:

(1) For each $n \in \mathbb{N}, U_{n} \cap V=\emptyset$ and $U_{n} \cap U_{m}=\emptyset$ if $n \neq m$.

(2) For each $n \in \mathbb{N}, U_{n} \cap \alpha\left(t_{0}\right) \neq \emptyset$.

(3) $\alpha\left(t_{0}\right) \subseteq V \cup\left(\bigcup\left\{U_{n}: n \in \mathbb{N}\right\}\right)$ and $B \subseteq V$. 
Let $U=V \cup\left(\bigcup\left\{U_{n}: n \in \mathbb{N}\right\}\right)$. Observe that $\alpha\left(t_{0}\right) \in\langle U\rangle$ and $t_{0}>0$. Since $\alpha$ is continuous there exists $\delta>0$ such that $\alpha\left(\left[t_{0}-\delta, t_{0}\right]\right) \subseteq\langle U\rangle$. Let $t_{1} \in\left[t_{0}-\delta, t_{0}\right] \cap T$. Since $\overline{\alpha\left(t_{1}\right)-B}$ is compact there exists a finite subset $F$ of $\mathbb{N}$ such that $\overline{\alpha\left(t_{1}\right)-B} \subseteq V \cup\left(\bigcup\left\{U_{n}: n \in F\right\}\right)$. Observe that $\alpha\left(\left[t_{0}-\delta, t_{0}\right]\right) \subseteq$ $2^{X}$ is connected. Let $N \in \mathbb{N}-F$. We have that $U_{N} \cap\left(\bigcup \alpha\left(\left[t_{0}-\delta, t_{0}\right]\right)\right)$ is a nonempty, open and closed subset of $\bigcup \alpha\left(\left[t_{0}-\delta, t_{0}\right]\right)$. Now, by Lemma 4.9, $\alpha(t) \cap U_{N} \neq \emptyset$, for each $t \in\left[t_{0}-\delta, t_{0}\right]$, in particular $\alpha\left(t_{1}\right) \cap U_{N} \neq \emptyset$. Since $B \subseteq V, U_{N} \cap V=\emptyset$ and $\alpha\left(t_{1}\right) \subseteq U$ we have that $\left(\overline{\alpha\left(t_{1}\right)-B}\right) \cap U_{N} \neq \emptyset$, a contradiction. This proves that $t_{0} \in T$. Now, since $1 \notin T$, we have that $t_{0}<1$. We assert that $\alpha\left(t_{0}\right)$ is not compact. Suppose that $\alpha\left(t_{0}\right)$ is compact and let $U$ be an open subset of $X$ such that $\alpha\left(t_{0}\right) \in\langle U\rangle$. Since $X$ is locally compact there exists an open subset $V$ of $X$ such that $\alpha\left(t_{0}\right) \subseteq V \subseteq \bar{V} \subseteq U$ and $\bar{V}$ is compact. There exists $\varepsilon>0$ such that $\alpha\left(\left(t_{0}, t_{0}+\varepsilon\right)\right) \subseteq\langle V\rangle$. Then, for each $t \in\left(t_{0}, t_{0}+\varepsilon\right)$ we have that $\alpha(t) \subseteq V \subseteq \bar{V}$. Since $\bar{V}$ is compact, $\alpha(t)$ is compact too, for each $t \in\left(t_{0}, t_{0}+\varepsilon\right)$. Now $\overline{\alpha(t)-B}$ is a closed subset of $\alpha(t)$, so $\overline{\alpha(t)-B}$ is compact, for each $t \in\left(t_{0}, t_{0}+\varepsilon\right)$, a contradiction. This proves that $\alpha\left(t_{0}\right)$ is not compact, consequently $\alpha\left(t_{0}\right)$ is infinite. We define $K=\overline{\alpha\left(t_{0}\right)-B}$. Observe that $\alpha\left(t_{0}\right)-K \subseteq B$, hence it is closed, discrete and countably infinite. Assume that $\alpha\left(t_{0}\right)-K=\left\{c_{n}: n \in \mathbb{N}\right.$ and $c_{n} \neq c_{m}$ if $\left.n \neq m\right\}$. Now, take a sequence $\left\{t_{k}\right\}_{k \in \mathbb{N}}$ of points in $[0,1]-T$ such that $\left\{t_{k}\right\}_{k \in \mathbb{N}}$ converges to $t_{0}$. Since $\alpha$ is continuous, we have that $\left\{\alpha\left(t_{k}\right)\right\}_{k \in \mathbb{N}}$ converges to $\alpha\left(t_{0}\right)$. Observe that $\overline{\alpha\left(t_{k}\right)-B}$ is not compact, for each $k \in \mathbb{N}$. Then $K, \alpha\left(t_{0}\right)$ and $B$ satisfy the conditions of Lemma 4.10. Thus there is an open subset of $X, V$, and there exists $M \in \mathbb{N}$ such that for each $m \in \mathbb{N}$ there exists an open subset of $X, U_{m}$, and there exists $n_{m} \in \mathbb{N}$ such that:

(1) The elements of $\left\{U_{m}: m \in \mathbb{N}\right\} \cup\{V\}$ are pairwise disjoint.

(2) $K \subseteq V$ and $c_{m} \in U_{m}$, for each $m \in \mathbb{N}$.

(3) $n_{m}<n_{m+1}$, for each $m \in \mathbb{N}$.

(4) For each $m \in \mathbb{N}$, there exists $x_{m} \in \alpha\left(t_{M+m}\right) \cap\left(U_{n_{m}}-\left\{c_{n_{m}}\right\}\right)$.

For each $m \in \mathbb{N}$, we define

$$
V_{m}=\left\{\begin{aligned}
U_{m}, & \text { if } m \neq n_{k}, \text { for each } k \in \mathbb{N} \\
U_{n_{k}}-\left\{x_{k}\right\}, & \text { if } m=n_{k}, \text { for some } k \in \mathbb{N} .
\end{aligned}\right.
$$

Let $W=V \cup\left(\bigcup\left\{V_{m}: m \in \mathbb{N}\right\}\right)$. Clearly $\alpha\left(t_{0}\right) \in\langle W\rangle$. Since $\left\{\alpha\left(t_{k}\right)\right\}_{k \in \mathbb{N}}$ converges to $\alpha\left(t_{0}\right)$, there exists $N \in \mathbb{N}$ such that $\alpha\left(t_{M+N}\right) \in\langle W\rangle$. Observe that $x_{N} \in \alpha\left(t_{M+N}\right) \cap\left(U_{n_{N}}-\left\{c_{n_{N}}\right\}\right)$. The fact that $U_{r} \cap U_{s}=\emptyset$, if $r \neq s$, implies that $x_{N} \in V_{n_{N}}=U_{n_{N}}-\left\{x_{N}\right\}$, a contradiction. Hence, there is no path from $A$ to $B$ in $T D(X)$.

Proposition 4.12. We define a relation $\sim$ on $\mathcal{P}(\mathbb{N})$ as follows: If $A, B \subseteq$ $\mathbb{N}$, we set $A \sim B$ if and only if there exists $N \in \mathbb{N}$ such that $A \cap[N, \infty)=$ $B \cap[N, \infty)$. The following conditions hold: 
(1) The relation $\sim$ is an equivalence relation.

(2) If $A, B \subseteq \mathbb{N}$ are finite, then $A \sim B$.

(3) The equivalence class $[A]_{\sim}$ is countably infinite, for each $A \in \mathcal{P}(\mathbb{N})$.

(4) $\sim$ has $\mathfrak{c}$ equivalence classes.

THEOREM 4.13. If $X$ is a locally compact, not compact, Hausdorff and Lindelöf space, then $T D(X)$ has at least $\mathfrak{c}$ path components.

Proof. Since $X$ is a Lindelöf space but it is not compact, we have that $X$ contains a discrete, countably infinite and closed subset, $C$. Assume that $C=\left\{x_{n}: n \in \mathbb{N}\right\}$ and let $N, M \subseteq \mathbb{N}$ such that $N$ is not related to $M$ in the sense of Proposition 4.12. We define $A=\left\{x_{n}: n \in M\right\}$ and $B=\left\{x_{n}: n \in\right.$ $N\}$. Without loss of generality assume that $A-B$ is infinite. By Theorem 4.11 we have that there is no path in $T D(X)$ from $A$ to $B$. Since this happens whenever $M, N \subseteq \mathbb{N}$ are such that $[M] \neq[N]$, from Proposition $4.12, \sim$ has $\mathfrak{c}$ equivalence classes. Consequently $T D(X)$ has at least $\mathfrak{c}$ path components.

COROLlary 4.14. If $X$ is locally compact, not compact, Hausdorff, second countable space, then $T D(X)$ has exactly $\mathfrak{c}$ path components.

Proof. Since $X$ has a base of cardinality $\omega$, then $X$ has at most $2^{\omega}$ open sets. In other words, $X$ has at most $\mathfrak{c}$ closed set. Thus $|T D(X)| \leq \mathfrak{c}$. The result follows from Theorem 4.13.

Corollary 4.15. If $X$ is a noncompact manifold, then $T D(X)$ has exactly $\mathfrak{c}$ path components.

Corollary 4.16. Let $X$ be a locally compact, Hausdorff and Lindelöf space. If $T D(X)$ is pathwise connected, then $X$ is compact.

We note that the converse of Corollary 4.16 is not true: for this it is enough to consider the dendroid of Example 4.5.

TheOrem 4.17. Let $X$ be a locally compact, Lindelöf and Hausdorff space. If $L$ and $M$ can be connected by a path in $T D(X)$, then $L$ is compact if and only if $M$ is compact.

Proof. Let $L, M \in T D(X)$ and let $\alpha:[0,1] \rightarrow T D(X)$ be a continuous function such that $\alpha(0)=L$ and $\alpha(1)=M$. It is enough to prove that if $L$ is compact, then $M$ is compact. Suppose that $L$ is compact and $M$ is not compact. Let $r=\sup \{t \in[0,1]: \alpha(t)$ is compact $\}$. We assert that $\alpha(r)$ is not compact. If $r=1$, then $\alpha(r)$ is not compact. Now, suppose that $r<1$ and $\alpha(r)$ is compact. Since $X$ is locally compact, there exists an open subset, $V$, of $X$ such that $\alpha(r) \subseteq V \subseteq \bar{V} \subseteq X$ and $\bar{V}$ is compact. Since $\alpha$ is continuous, there exists $\varepsilon>0$ such that $\alpha((r, r+\varepsilon)) \subseteq\langle V\rangle$. Consequently $\alpha(t)$ is compact, for each $t \in(r, r+\varepsilon)$, a contradiction. Therefore $\alpha(r)$ is not compact and so $r>0$. On the other hand, since $X$ is a Lindelöf space and 
$\alpha(r)$ is a closed subset of $X$, we have that $\alpha(r)$ is a Lindelöf space. So $\alpha(r)$ is not countably compact, consequently $\alpha(r)$ contains a closed, discrete and countably infinite subset $A$. Assume that $A=\left\{x_{n}: n \in \mathbb{N}\right\}$. Now, for each $n \in \mathbb{N}$ there is an open subset $V_{n}^{\prime}$ of $X$ such that $x_{n} \in V_{n}^{\prime}$ and $V_{n}^{\prime} \cap V_{m}^{\prime}=\emptyset$ if $n \neq m$. We know that $\alpha(r)$ is 0 -dimensional, then for each $n \in \mathbb{N}$ there is an open-closed subset $W_{n}$ of $\alpha(r)$ such that $x_{n} \in W_{n} \subset V_{n}^{\prime}$. By Lemma 4.6 there exists a cellular family $\left\{V_{n}: n \in \mathbb{N}\right\}$ of open-closed subsets of $\alpha(r)$ such that $x_{n} \in V_{n} \subset W_{n} \subset V_{n}^{\prime}$ and $\bigcup\left\{V_{n}: n \in \mathbb{N}\right\} \in 2^{\alpha(r)} \subset 2^{X}$. Note that the family $\left\{V_{n}: n \in \mathbb{N}\right\} \cup\left\{\alpha(r)-\bigcup\left\{V_{n}: n \in \mathbb{N}\right\}\right\}$ satisfies the conditions of Lemma 4.7, so there exists a cellular family $\left\{U_{n}: n \in \mathbb{N} \cup\{0\}\right\}$ in $X$ such that $\left(\alpha(r)-\bigcup\left\{V_{n}: n \in \mathbb{N}\right\}\right) \subseteq V_{0}$ and for each $n \in \mathbb{N}, V_{n} \subseteq U_{n}$. Observe that $\alpha(r) \subseteq \bigcup\left\{U_{n}: n \in \mathbb{N} \cup\{0\}\right\}$, i.e. $\alpha(r) \in\left\langle\bigcup\left\{U_{n}: n \in \mathbb{N} \cup\{0\}\right\}\right\rangle$. Since $\alpha$ is continuous, there is $\varepsilon>0$ such that $\alpha([r-\varepsilon, r+\varepsilon]) \subseteq\left\langle\bigcup\left\{U_{n}: n \in \mathbb{N} \cup\{0\}\right\}\right\rangle$. Take $t_{0} \in[r-\varepsilon, r)$ such that $\alpha\left(t_{0}\right)$ is compact. So there exists $N \in \mathbb{N}$ such that $\alpha\left(t_{0}\right) \cap U_{N}=\emptyset$. Since $(\bigcup \alpha([r-\varepsilon, r+\varepsilon])) \cap U_{N}=(\bigcup \alpha([r-\varepsilon, r+\varepsilon])) \cap \bar{U}_{N}$ we have that $(\bigcup \alpha([r-\varepsilon, r+\varepsilon])) \cap U_{N}$ is a nonempty open and closed subset of $\bigcup \alpha([r-\varepsilon, r+\varepsilon])$. Since $\alpha([r-\varepsilon, r+\varepsilon])$ is a connected subset of $2^{X}$, by Lemma 4.9, we have that $\alpha(t) \cap\left((\bigcup \alpha([r-\varepsilon, r+\varepsilon])) \cap U_{N}\right) \neq \emptyset$, for each $t \in[r-\varepsilon, r+\varepsilon]$, so $\alpha\left(t_{0}\right) \cap U_{N} \neq \emptyset$, a contradiction. This proves that $M$ is compact.

The next Theorem can be proved in the same way as Theorem 3.2 of [4].

TheOrem 4.18. If $X$ is a Hausdorff space, $\alpha:[0,1] \rightarrow T D(X) \cap K(X)$ is a path and $p \in \alpha(0)$, then there exists a continuous function $f:[0,1] \rightarrow X$ such that $f(0)=p$ and $f(t) \in \alpha(t)$ for each $t \in[0,1]$.

THEOREM 4.19. If $X$ is a locally compact, Lindelöf and Hausdorff space such that $T D(X)$ is pathwise connected, then $X$ is pathwise connected.

Proof. Let $x, y \in X$ and $\alpha:[0,1] \rightarrow T D(X)$ be a path such that $\alpha(0)=\{x\}$ and $\alpha(1)=\{y\}$. Note that $\{x\}$ is compact, so by Theorem 4.17 we have that $\alpha([0,1]) \subseteq T D(X) \cap K(X)$. Now, by Theorem 4.18 there exists a path $\gamma:[0,1] \rightarrow X$ such that $\gamma(0)=x$ and $\gamma(1) \in\{y\}$.

Note that the converse of Theorem 4.19 is not true, for this it is enough to consider the dendroid of Example 4.5.

ACKNOWLEDGEMENTS.

We are thankful to the referee for useful suggestions which helped to improve this paper.

\section{REFERENCES}

[1] J. J. Charatonik, Two invariants under continuity and the incomparability of fans, Fund. Math. 53 (1963/64), 187-204.

[2] J. J. Charatonik and C. Eberhart, On smooth dendroids, Fund. Math. 67 (1970), 297-322. 
[3] S. García-Ferreira and Y. F. Otriz-Castillo, The hyperspace of convergent sequences, Topol. Appl. 196 (2015), 795-804.

[4] S. García-Ferreira and R. Rojas Hernández, Connectedness like properties on the hyperspace of convergent sequences, Topol. Appl. 230 (2017), 639-647.

[5] A. Illanes and S. B. Nadler, Jr., Hyperspaces. Fundamental and recent advences, Marcel Dekker, Inc., New York, 1999.

[6] J. M. Martinez-Montejano, Mutual aposyndesis of symmetric products, Topology Proc. 24 (1999), 203-213.

[7] D. Maya, P. Pellicer-Covarrubias and R. Pichardo-Mendoza, General properties of the hyperspace of convergent sequences, Topology Proc. 51 (2018), 143-168.

[8] E. Michael, Topologies on spaces of subsets, Trans. Amer. Math. Soc. 71 (1951), $152-182$.

[9] L. Mohler, A characterization of smoothness in dendroids, Fund. Math. 67 (1970), 369-376.

[10] S. B. Nadler, Jr., Hyperspaces of sets, Marcel Dekker, Inc., New York, 1978.

[11] S. B. Nadler, Jr., Continuum theory. An introduction, Marcel Dekker, Inc., New York, 1992.

[12] G. T. Whyburn, Analytic topology, Amer. Math. Soc. New York, 1942.

R. Escobedo

Facultad de Ciencias Físico-Matemáticas

Benemérita Universidad Autónoma de Puebla

Av. San Claudio y 18 sur, Col. San Manuel, Edificio FM3-210

Ciudad Universitaria C.P. 72570, Puebla

México

E-mail: escobedo@fcfm.buap.mx

P. Pellicer-Covarrubias

Departmento de Matemáticas

Universidad Nacional Autónoma de México

Circuito Exterior, Ciudad Universitaria, Ciudad de México, C.P. 04510

México

E-mail: paty@ciencias.unam.mx

V. Sánchez-Gutiérrez

Facultad de Ciencias Físico-Matemáticas

Benemérita Universidad Autónoma de Puebla

Av. San Claudio y 18 sur, Col. San Manuel, Edificio FM3-210

Ciudad Universitaria C.P. 72570, Puebla

México

E-mail: rompc190787@gmail.com

Received: 11.4.2019.

Revised: 31.8 .2019$. 